\title{
Chitons (Mollusca: Polyplacophora) from the Venezuelan Coasts, Southern Caribbean: A Checklist and Brief Review in Advance of Their Knowledge
}

\author{
Ignacio Agudo-Padrón*
}

Geographer \& Research Malacologist, CEO Project “Avulsos Malacológicos - AM, Brazil

*Corresponding author: Dr. Ignacio Agudo-Padrón, Geographer \& Research Malacologist, CEO Project “Avulsos Malacológicos - AM”, Caixa Postal (P.O.Box) 010, 88010-970 Centro, Florianópolis, Santa Catarina/SC, Brazil; Email: ignacioagudo@gmail.com

Received: December 31, 2020; Accepted: January 06, 2021; Published: January 16, 2021

\section{Introduction}

Chitons or polyplacophores constitute a of the eight classes of the filum Mollusca. They are generally small, flattened and elongated animals, provided with eight (8) overlapping dorsal plates or valves, bordered by a belt of scales and/or calcareous spicules formed by the mantle, called perinotum. The cavity of the mantle encloses the foot, which is expands forming a large sole, which not only serves for the locomotion but also to maintain firm contact with the surface of the substrates where they live (Gracia C. et al. 2005: 117) (Figure 1).

In relation to the taxonomic Class Polyplacophora, some 850 recent species of Chitons are known, most of which live in the Western Pacific and the Western coast of Central America, while in the Caribbean and the European Mediterranean they are more poorly represented (Tejeda et al. 2015: 112), being that in the essential pioneering contribution of Kaas (1972) 34 species were already reported for the Caribbean and the Gulf of Mexico.

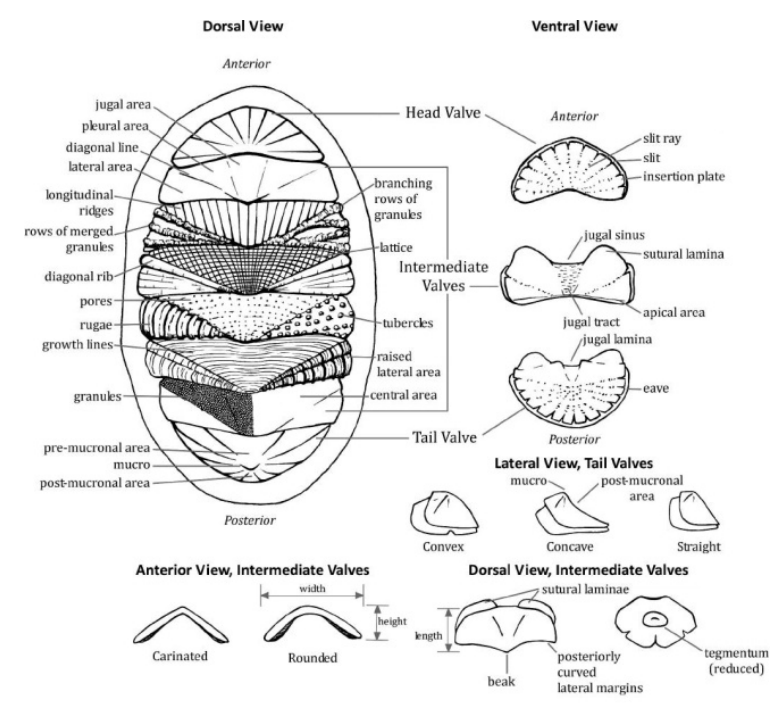

\section{Background}

A general synthesis about the current knowledge of the shallow water polyplacophoran molluscs (chitons) occurring in the Southern Caribbean of Venezuela is presented, involving bibliographical revision/database (summarized informations [1] from the "Northeast" region, coastal \& insular, and [2] from "Los Roques Archipelago Marine National Park"), and historical rescue of informations (field notebooks) which includes inedit "personal report" of manual collection of specimens (total of 17 specific forms) in three (3) coastal areas (Northeast (CaA) - Sucre State $\left(\sim 10^{\circ} 3844^{\prime \prime} \mathrm{N}\right.$ \& 6302' 20"W) in "Golfo de Cariaco" (Cariaco Gulf) region, with 12 species; Central (CaB) - Vargas (“Catia La Mar" sector 10³6’ N \& $67^{\circ} 02^{\prime} \mathrm{W}$ ) \& Miranda ("Los Totumos/Higuerote" sector $\sim 10^{\circ} 29^{\prime} 00 \mathrm{~N}$ \& 66 $\left.06^{\circ} 00^{\prime \prime} \mathrm{W}\right)$ States, with 14 species; and Central Western $(\mathrm{CaC})$ -

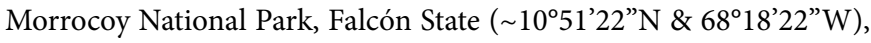
with 9 species) (Figure 2 - Map \& Table 1) of the country between the years 1976 and 1989, parallel to field studies of cnidarian fauna - sea anemones [3].

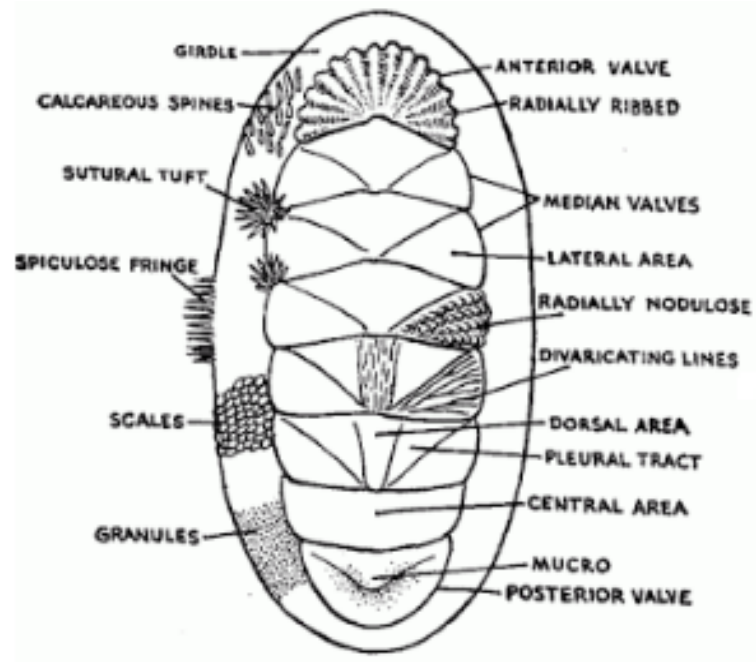

Figure 1: Structural morphology of Chitons. Source: GOOGLE, Creative Commons. 


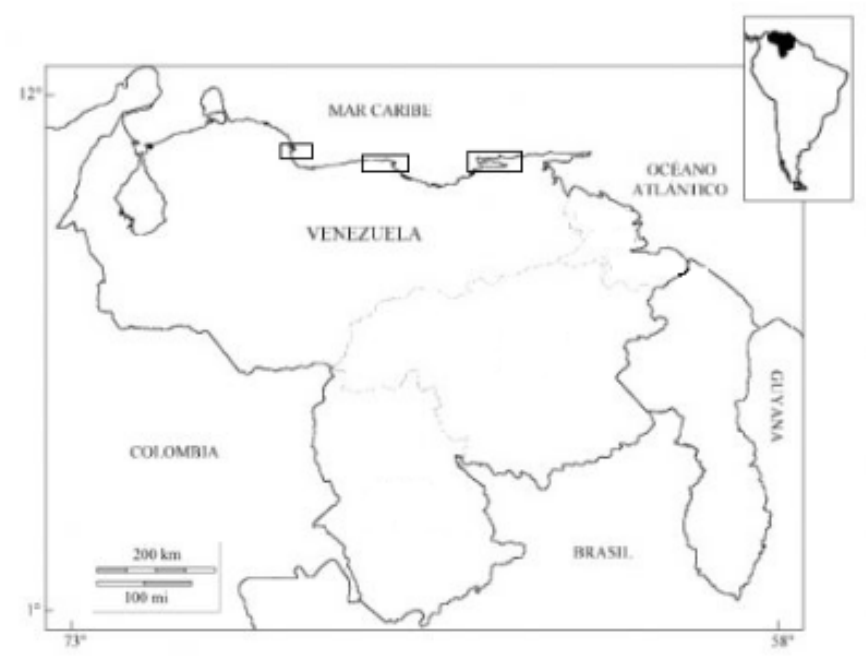

Figure 2: Venezuelan coastal areas (rectangles) where manual collections of polyplacophores (chitons) were carried out by us between the years 1976 and 1989: Northeast region - CaA (right angle), Central region - $\mathrm{CaB}$ (center angle), Central Western region - $\mathrm{CaC}$ (left angle). Credit Map: Original by A. Ignacio Agudo-Padrón, Project AM.

Unfortunately, the samples were "lost" in the course of a catastrophic event (flood), before it can be formally deposited in a scientific institution/museum (... this collection also included species obtained during the period in several coastal insular and mainland localities \& representative marine environments of the country ... an unhappy, irreparable and heartbreaking loss !!).

Counting up still, recently (since the year 2014), with the invaluable participatory assistance and support of "Northeast" (Sucre State) local researcher ecologists, naturalists and collaborating informants (Bello-Pulido et al. 2016), with field collections carried out during the years 2015 and 2016, totalling 36 geographical sampling points, distributed in five (5) large predominantly rocky coastal bioregions, taxonomically involving five (5) families, eight (8) genera and eleven (11) certain species (Figure 3).

\section{Results \& Conclusions}

A updated checklist/inventory including a confirmed general total of 25 species, taxonomically distributed in 12 genera and six (6) families, complements the present brief report (Table 1), which makes it possible to make important preliminary comparisons with the polyplacophore fauna of other important Caribbean regions, such as Colombia ([4], with 22 species), Puerto Rico ([5], with 29 recognized species $\sim$ the place with the greatest diversity of polyplacophores in the region), the Hispaniola Island ([6], with 23 known species) and, most recently, Cuba ([7], with 27 species).

Particularly, the Caribbean/eastern surf chiton species CALLISTOPLACIDAE Ceratozona squalida (C. B. Adams, 1845) (Figure 3 and Table 1) was one of the first relevant species recorded in the field by us in Venezuela (June 18 1982), with several specimens found in the Northeast region - CaA ("Playa San Luis" < San Luis Beach $>$ sector, Cumaná city 10 $27^{\prime} 00^{\prime} \mathrm{N} \& 64^{\circ} 10^{\prime} 00^{\prime}$ W, Sucre State) in the rubble of a ramp/dock of cement \& stones half-buried on sands
Table 1: Checklist of the Chitons (Mollusca: Polyplacophora) known today for Venezuela (Southern Caribbean) and distribution on the coastal areas "previously verified in field" (Figure 2).

\begin{tabular}{|c|c|c|c|}
\hline Detected Species & $\mathrm{CaA}$ & $\mathrm{CaB}$ & $\mathrm{CaC}$ \\
\hline \multicolumn{4}{|l|}{ Class Polyplacophora Gray, 1821} \\
\hline \multicolumn{4}{|l|}{ Subclass Neoloricata Bergenhayn, 1955} \\
\hline \multicolumn{4}{|l|}{ Order Chitonida Thiele, 1909} \\
\hline \multicolumn{4}{|l|}{ Suborder Acanthochitonina Bergenhayn, 1930} \\
\hline \multicolumn{4}{|l|}{ Family Acanthochitonidae Pilsbry, 1893} \\
\hline \multicolumn{4}{|l|}{ Americhiton andersoni (Watters, 1981) } \\
\hline \multicolumn{4}{|l|}{ Americhiton belesae (Abbott, 1954) } \\
\hline Acanthochitona hemphilli (Pilsbry, 1893) & & $\mathrm{X}$ & \\
\hline Acanthochitona pygmaea (Pilsbry, 1893) & $\mathrm{X}$ & & \\
\hline \multicolumn{4}{|l|}{ Acanthochitona retrojecta (Pilsbry, 1894) } \\
\hline \multicolumn{4}{|l|}{ Acanthochitona rhodea (Pilsbry, 1893) } \\
\hline \multicolumn{4}{|l|}{ Acanthochitona venezuelana Lyons, 1988} \\
\hline \multicolumn{4}{|l|}{ Suborder Chitonina Thiele, 1909} \\
\hline \multicolumn{4}{|l|}{ Family Chaetopleuridae Plate, 1899} \\
\hline Chaetopleura apiculata (Say, 1834) & $\mathrm{X}$ & & \\
\hline \multicolumn{4}{|l|}{ Family Lepidochitonidae Iredale, 1914} \\
\hline Lepidochitona liozonis (Dall \& Simpson, 1901) & & $\mathrm{X}$ & $\mathrm{X}$ \\
\hline \multicolumn{4}{|l|}{ Lepidochitona sp 1} \\
\hline \multicolumn{4}{|l|}{ Lepidochitona sp 2} \\
\hline \multicolumn{4}{|l|}{ Family Chitonidae Rafinesque, 1815} \\
\hline Acanthopleura granulata (Gmelin, 1791) & $\mathrm{X}$ & $\mathrm{X}$ & $\mathrm{X}$ \\
\hline Chiton marmoratus Gmelin, 1791 & $\mathrm{X}$ & $\mathrm{X}$ & $\mathrm{X}$ \\
\hline Chiton squamosus Linnaeus, 1764 & $\mathrm{X}$ & $\mathrm{X}$ & \\
\hline Chiton viridis Spengler, 1797 & $\mathrm{X}$ & & \\
\hline Leptochiton cancellatus (G. B. Sowerby II, 1840) & $\mathrm{X}$ & $\mathrm{X}$ & $\mathrm{X}$ \\
\hline Rhyssoplax janeirensis (Gray, 1828) & $\mathrm{X}$ & & \\
\hline \multicolumn{4}{|l|}{ Family Callistoplacidae Pilsbry, 1893} \\
\hline Callistochiton portobelensis Ferreira 1976 & & $\mathrm{X}$ & \\
\hline Ceratozona squalida (Adams, 1845) & $\mathrm{X}$ & $\mathrm{X}$ & $\mathrm{X}$ \\
\hline \multicolumn{4}{|l|}{ Family Ischnochitonidae Dall, 1899} \\
\hline Ischnochiton erythronotus (C.B. Adams, 1845) & $\mathrm{X}$ & $\mathrm{X}$ & $\mathrm{X}$ \\
\hline \multicolumn{4}{|l|}{ Ischnochiton hartmeyeri Thiele, 1916} \\
\hline Ischnochiton papillosus (C.B. Adams, 1845) & $\mathrm{X}$ & $\mathrm{X}$ & $\mathrm{X}$ \\
\hline Ischnochiton striolatus (Gray, 1829) & $\mathrm{X}$ & $\mathrm{X}$ & $\mathrm{X}$ \\
\hline Stenoplax boogii (Haddon, 1886) & & $\mathrm{X}$ & \\
\hline Stenoplax purpurascens (Haddon, 1886) & $\mathrm{X}$ & $\mathrm{X}$ & $\mathrm{X}$ \\
\hline
\end{tabular}

Abbreviations: CaA: Northeast Area; $\mathrm{CaB}$ : Central Area; $\mathrm{CbC}$ : Central Western Area. Source informations: Archive/DataBase of the "Project AM".

of the coastal urban area - tides zone, encrusted with algae, together with a beautiful specimen of caribbean branching anemone Lebrunia neglecta (- danae) Duchassaing \& Michelotti, 1860 (see GonzálezMuñoz et al. 2016: 29 - Appendix 2, Table 3, "record no. 25”) [8].

At least a one very little/diminute chiton species with tufts of glasshair-like bristles - ACANTHOCHITONIDAE cf. Acanthochitona andersoni Watters, 1981, Acanthochitona venezuelana Lyons, 1988 or Acanthochitona pygmaea (Pilsbry, 1893) - exist in the environment 


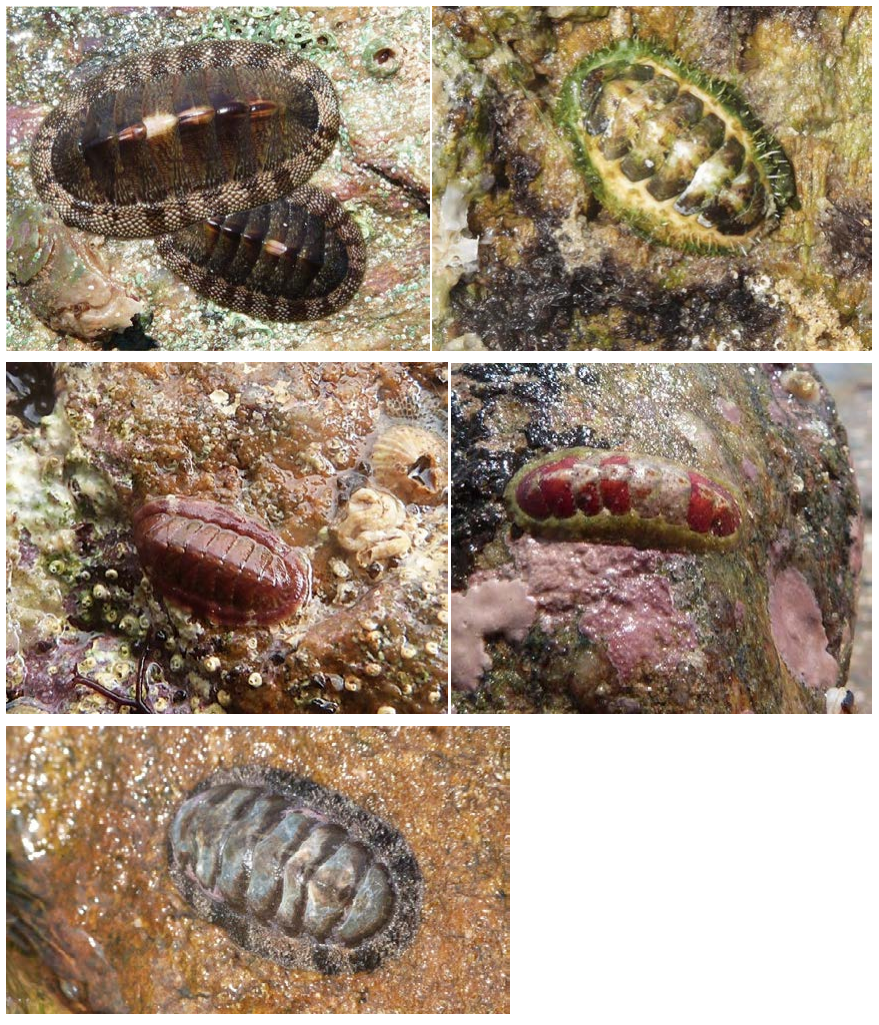

Figure 3: Shallow water polyplacophoran (chitons) biodiversity in Northeast coastal region of Venezuela: CHITONIDAE Leptochiton cancellatus (G. B. Sowerby II, 1840), CALLISTOPLACIDAE Ceratozona squalida (Adams, 1845), LEPIDOCHITONIDAE Lepidochitona liozonis (Dall \& Simpson, 1901), ISCHNOCHITONIDAE Stenoplax purpurascens (Haddon, 1886), CHITONIDAE Acanthopleura granulata (Gmelin, 1791). (Photos: Jesús Antonio Bello-Pulido, collaborator of the "Project AM").

(reef substrate, loose dead coral) of the restrict "Refúgio de Fauna Silvestre Isla de Aves" (Bird Island Wildlife Refuge 1541'00”N \& $\left.63^{\circ} 37^{\prime} 00^{\prime \prime} \mathrm{W}\right)$, Northern sector of the Venezuelan Caribbean Sea, occurrence that still needs to be properly investigated.
Among other potential forms still pending confirmation in the country, highlights the iconic species CHITONIDAE Tonicia schrammi (Shuttleworth, 1856), the "gold-flecked chiton", typical representative of the Caribbean Polyplacophoran fauna < http://www.marinespecies.org/photogallery.php?album $=704 \&$ pic $=103379 ;$ https:// www.conchology.be/?t=68\&u=1096898\&g=97858aa8e0d2e81d7c41f$5884 \mathrm{~d} 1 \mathrm{fc} 0 \mathrm{c} 4 \& \mathrm{q}=0 \mathrm{a} 23 \mathrm{a} 7694181 \mathrm{e} 63 \mathrm{fa} 3 \mathrm{a} 9 \mathrm{c} 05450 \mathrm{ac6178}>$.

Finally, all the species listed in the present contribution (Table 1) were previously revised through the global platform "WoRMS World Register of Marine Species" < http://www.marinespecies.org/ $>$, checking your current taxonomic status/situation.

\section{References}

1. Capelo JC, Buitrago J (1998) Distribución geográfica de los moluscos marinos en el Oriente de Venezuela. Memória 58: 109-160.

2. Jiménez M, Allen T, Fernández J, Narciso S (2014) Moluscos asociados al coral Montastrea annularis em el Parque Nacional Archipiélago de Los Roques. Acta Biol. Venez 34: 233-243.

3. González-Muñoz R, Simões N, Guerra-Castro EJ, Hernández-Ortíz C, Carrasquel $\mathrm{G}$ et al. (2016) Sea anemones (Cnidaria: Actiniaria, Corallimorpharia, Ceriantharia, Zoanthidea) from marine shallow-water environments in Venezuela: new records and an updated inventory. Marine Biodiversity Records: 9.

4. Gracia CA, Díaz JM, Ardila NE (2005) Quitones (Mollusca: Polyplacophora) del Mar Caribe Colombiano. Biota Colombiana 6: 117-125.

5. Garcia-Rios CI (2005) Los quitones (Mollusca: Polyplacophora) em Puerto Rico. Santo Domingo, República Dominicana: Universidad Autónoma de Santo Domingo, V Congreso de Biodiversidad Caribeña, Volume V, Conference paper.

6. Herrera-Moreno A, Fernández LB (2010) Lista de espécies de quitones (Mollusca: Polyplacophora) conocidas para la Hispaniola. Novitates Caribaea 3: 62-68.

7. Tejeda CR, Maceira1 D, Cedar García-Ríos C, Espinosa J (2015) Listado actualizado y claves para Polyplacophora (Mollusca) en Cuba. Novitates Caribaea 8: 112-119.

8. Kaas P (1972) Polyplacophora of the Caribbean region. Studies on the Fauna of Curacao and other Caribbean Islands 137: 1-162. 